\title{
Does the duration of infertility affect semen parameters and pregnancy rate after varicocelectomy? A retrospective study
}

\author{
Mohammed A. Al-Ghazo, Ibrahim Fathi Ghalayini, Rami S al-Azab, Ibrahim Bani-Hani, \\ Mohammad S. Daradkeh
}

King Abdullah university Hospital, faculty of Medicine, Jordan University of Science and technology, Irbid-Jordan

\begin{abstract}
Objectives: The most common indication for treatment of varicocele is still male subfertility. The aim of this study was to explore the effect of infertility duration on semen parameters and spontaneous pregnancy rate after varicocelectomy. Materials and Methods: The medical records of 183 infertile patients with clinical varicocele were retrospectively reviewed. The patients were divided into three groups according to the duration of infertility (group I, 1-3 years, group II, 3-6 years and group III, > 6 years). Total sperm motility counts (TMCs) before and after varicocelectomy and spontaneous pregnancy rate among these groups were statistically compared.

Results: The greatest changes, regarding preoperative and postoperative TMCs and spontaneous pregnancy rate were noticed between group I and III. Preoperative TMCs in group I and III was $15.2 \pm 1.2,7.8 \pm 1.4$, respectively (p $<0.05$ ). Postoperative TMCs in group I and III was $33.7 \pm 2.5,25.2 \pm 1.9$, respectively $(\mathrm{p}<0.05)$. An overall spontaneous pregnancy rate of $34.4 \%$ was achieved after inguinal varicocelectomy. The greatest spontaneous pregnancy rate was achieved in Group I (37.3\%), and the lowest pregnancy rate in Group III $(26.3 \%)(\mathrm{P}<0.05)$.

Conclusions: Surgical varicocelectomy improves the total sperm motility counts especially in patients who have a TMCS more than 5 million and improves the spontaneous pregnancy rates. The improvement in the spontaneous pregnancy rates after varicocelectomy correlates negatively with the duration of infertility. Therefore, duration of infertility should be considered in treating a patient with a varicocele as a cause of infertility.
\end{abstract}

Key words: infertility; spermatogenesis; varicocele; semen analysis

Int Braz J Urol. 2011; 37: 745-750

\section{INTRODUCTION}

Infertility, defined as the inability to conceive after one year of unprotected intercourse, affects $15 \%$ of couples (1). The most common identifiable cause of male infertility (subfertility) is a varicocele, a condition of palpably distended veins of the pampiniform plexus of the spermatic cord $(2,3)$. Varicoceles occur in approximately $15 \%$ of the general male population but are more common (25\%-35\% prevalence) in infertile men and are the most common physical abnormality in infertile men $(3,4)$. Various mechanisms have been proposed to explain infertility in men with varicocele. These include hypoxia, stasis, testicular venous hypertension, elevated testicular temperature, increase in spermatic vein catecholamine, and increased oxidative stress (5). However, there have still been some undefined mechanisms of varicocele since some of the patients with varicocele have normal semen parameters (6). The most common indication for treatment of varicocele is still the male subfertility. The other possible indications are varicocele-related scrotal pain or swelling not relieved by conservative treatment. Duration and severity of varicocele may be a risk factor for infertility related with age 
in patients with varicocele (7). The mean time for semen improvement and spontaneous pregnancy after surgery is approximately five to seven months, respectively (8). However, the aetiology of underlying infertility is especially uncertain in men whose semen parameters do not significantly improve or do not achieve pregnancy following varicocele surgery. Marcello et al. (9) reported that high-grade varicocele (grade III), normal FSH, total motility over $60 \%$ and total motile sperm count over $5 \times 10^{6}$ (before surgery) are good prognostic factors of varicocele repair outcomes in infertile patients, while subclinical varicocele, presence of Y chromosome microdeletions, testicular atrophy and total motile sperm count less than $20 \times 10^{6}$ (after surgery) are poor prognostic factors. Zorba et al. (10) reported that the duration of infertility should be considered as another predictive factor for a positive seminal response to varicocelectomy and achieving pregnancy. It is uncertain the effect of varicocele surgery on the duration of infertility and outcome especially semen characteristics and pregnancy rate, only a limited number of studies have been reported and this subject is not completely understood.

The aim of this study is to evaluate the duration of infertility on postvaricocelectomy semen parameters and spontaneous pregnancy rates.

\section{MATERIALS AND METHODS}

Our institution's protocol review board approved this retrospective study.

From March 2003 to October 2009, we retrospectively reviewed the medical records of 183 patients, who underwent inguinal varicocelectomy because of infertility at King Abdullah University Hospital. Patients who were treated by other approaches such as high ligation, laparoscopic, or subinguinal microscopic surgery were excluded to avoid its effect on the results. The data collected included the following: age, period of infertility, spontaneous pregnancy, semen analysis before and after surgery, and complications and hormone profile. According to the period of infertility, 183 patients were divided into three groups. Group I 1-3 years, $(\mathrm{n}=102)$; group II, 4-6 years $(n=43)$, and group III, $>6$ years $(\mathrm{n}=38)$. Diagnosis of varicocele was made by physical examination of all patients. Varicoceles are graded as subclinical (impalpable, detected by ultrasonography), grade I (palpable by Valsava manoeuvre when upright), grade II (palpable without Valsava manoeuvre when upright), or grade III (visible) (11). Semen for analysis was obtained by masturbation after 3-5 days of sexual abstinence and was processed within one hour of ejaculation. All men had at least two semen analyses before and two semen analyses after surgery. We used the average data both preoperatively and postoperatively. All analyses were performed in the same laboratory by the same technicians. After liquefaction of semen, sperm concentration and motility were evaluated according to the World Health Organization (WHO) guidelines (12). The preoperative and postoperative total motile sperm counts (TMCs) were calculated by the following formula: $\mathrm{TMC}=$ ejaculate volume $(\mathrm{mL}) \times$ concentration $\left(\mathrm{x} 10^{6} / \mathrm{mL}\right) \times$ motile fraction (13). -Follicle-stimulating hormone (FSH), luteinizing hormone ( $\mathrm{LH})$, and testosterone were determined before varicocelectomy. Patients with abnormal hormone levels were excluded from the study. The spouses of these men were evaluated by the gynaecologists for factors related to female infertility, and patients whose spouses might have had concomitant female factors for infertility were also excluded. The mean follow-up period was 38 months (range, 14 to 82). During the follow-up period, we focused on surgical complications, varicocele recurrence, seminal fluid parameters and spontaneous pregnancy. All statistical analyses were performed with Statistical Package for the Social Sciences (SPSS), version 16.0.

Multivariate analysis was performed using age of the patient, grade of varicocele and duration of infertility in relation to spontaneous pregnancy rate. One-way analysis of variance and student's test were used to compare the preoperative and postoperative TMCs and pregnancy rates among the three groups. Probability $(\mathrm{P})$ values $<0.05$ were considered significant.

\section{RESULTS}

The mean age of patients included in this study was $28.4 \pm 5.4$ years (range 22-56). The mean age of patients in Groups I-III was $29.4 \pm 5.2$ (range 
22-46), $31.2 \pm 4.8$ (range 26-51), and $32.4 \pm 4.2$ (range 25-55), respectively, with $\mathrm{p}>0.05$.

Patients with unilateral (left-sided) and bilateral varicocele and clinical grades who underwent varicocelectomy for treatment of infertility are shown in Figure-1.

Abnormality in the total sperm motility count (TMCs) and pregnancy rates in patients among the three groups is shown in Table-1.
A total of 23 patients had severe oligozoospermia $<5 \mathrm{mil} / \mathrm{mL}$ and seven had azoospermia. The number of patients with azoospermia in Groups I-III was 4,2 , and 1, respectively. None of these patients could father a child after varicocelectomy. One azoospermic patient in Group I showed few motile sperms in the ejaculate after surgery, but he refused assisted reproduction for religious reasons. The number of patients with severe oligozoosper-

Figure 1 - Patents who underwent varicocelectomy for infertility treatment.

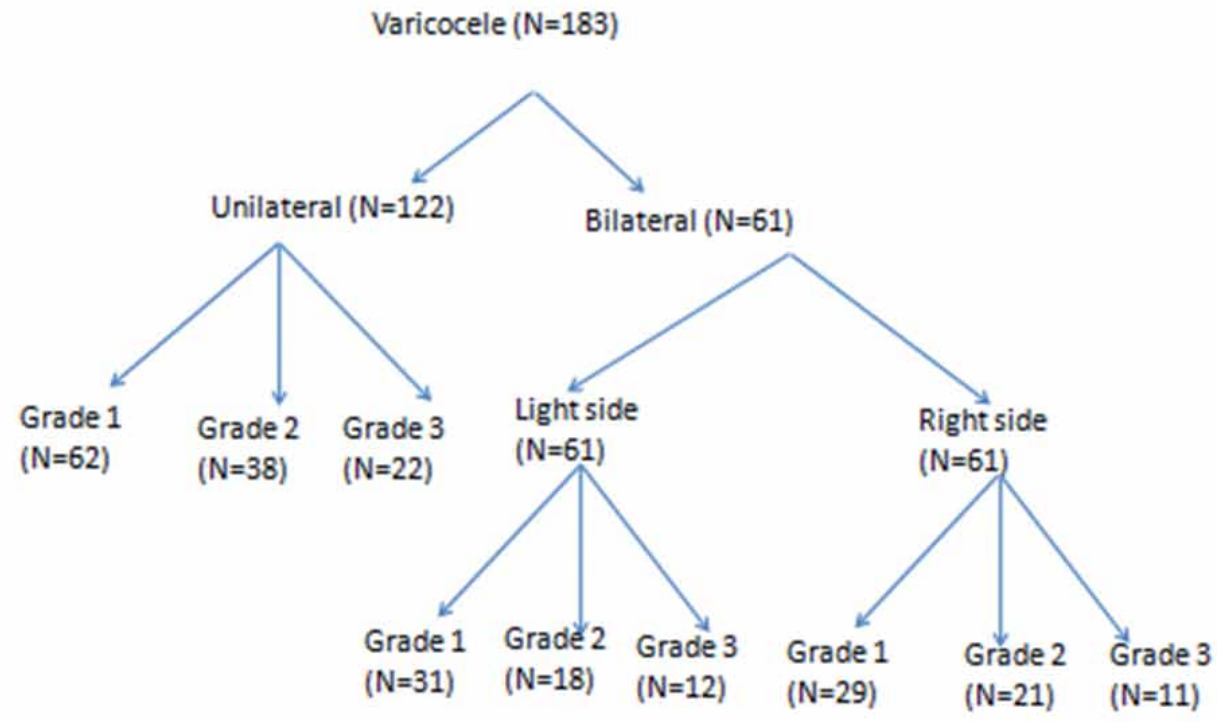

Table 1 - Preoperative and postoperative mean total sperm motility count and pregnancy rate between the three groups of patients.

\begin{tabular}{lccc}
\hline Variable & Group I & Group II & Group III \\
\hline Number of patients & 102 & 43 & 38 \\
Preoperative TMCs $\left(\mathrm{X} 10^{\circ}\right)$ & $15.6 \pm 1.2$ & $12.4 \pm 1.4$ & $8.0 \pm 1.7^{*}$ \\
Postoperative TMCs $\left(\mathrm{X} 10^{6}\right)$ & $33.7 \pm 2.5$ & $29.6 \pm 3.2$ & $25.2 \pm 1.9^{*}$ \\
Increase in the mean & 18.1 & 17.3 & 16.5 \\
TMCs(X10 & & & \\
Pregnancy rate\% & $37.3 \%$ & $34.9 \%$ & $26.3 \%^{*}$
\end{tabular}

* - Statistically significant difference between group 1 and 3

Group I: duration of infertility 1-3 years, Group II: duration of infertility 3-6 years, Group III: duration of infertility $>6$ years. 
mia in Groups I-III was 12, 4, and 7, respectively. The pregnancy rate in these groups with severe oligospermia was 2,0 , and 0 .

Changes in sperm motility rate in all groups before and after varicocelectomy are shown in Table-1. The greatest increase in motility rate after surgery was noted in group I $\left(17.2 \pm 1.4 \times 10^{6}\right)$, while patients in group III had the lowest increase in motility rate $\left(7.9 \pm 1.8 \times 10^{6}\right)$. The difference was statistically significant $(\mathrm{p}<0.05)$.

Preoperative motility rate had the tendency to decrease with an increasing period of infertility. The difference was not significant between Groups I and II or between Groups II and III ( $p>0.05)$, while it was significant between Group I and III $(p<0.05)$.

Spontaneous pregnancy was achieved in 63 out of 183 patients (34.4\%) after an average of 11.2 \pm 2.8 months after surgery (range 4-26 months). Most of these were achieved within the first year, in 57 of the 63 patients $(90.5 \%)$. No significant difference $(p>0.05)$ was observed among the groups in the time elapsed after surgery to pregnancy state. Pregnancy rates were $37.3 \%$. $34.9 \%$ and $26.32 \%$ in Groups I-III, respectively. The only statistically significant difference in spontaneous pregnancy rate was observed between Groups I and III $(p<0.05)$. Multivariate analysis revealed that the duration of infertility was the only significant factor affecting the pregnancy rate.

\section{DISCUSSION}

Varicocele, which is defined as an abnormal dilation of the testicular veins and pampiniform plexus, is the most frequent curable cause of male infertility (1). Deregulation of nitric oxide, reactive oxygen species, and regulators of apoptosis have been implicated in the pathophysiology of varicocele $(11,14)$. Several mechanisms may be involved in the negative effect of varicocele on male infertility. These include elevated intrascrotal temperature, reflux of adrenal and renal metabolites through renal vein, hypoxia, and ischemia $(15,16)$. A statistically significant deterioration in sperm count and motility throughout the follow-up period of untreated varicocele has been previously documented (17). More importantly, histological changes and atrophy have been demonstrated in the testes associated with varicocele (18). In this study, the difference in the preoperative sperm motility between Groups I and III was statistically significant $(\mathrm{p}<0.05)$. Moreover, we noticed that the sperm parameters correlate negatively with the increased period of infertility. These findings are in agreement with the results of Chehval and Prucell (17) who demonstrated a decline in sperm parameters over time in men with untreated varicocele. Many prospective studies have shown that testicular volume either fails to increase or actually decreases in testes that are associated with varicoceles $(19,20)$. Findings from previous studies provide strong evidence supporting the hypothesis that varicoceles exert a progressive deleterious effect on the testis during adolescence (17).

In the present study, we found a good response after varicocelectomy in the semen parameters in all patients with TMCs $>5 \times 10^{6}$ and the greatest improvement was found in patients in Group I, who had the greatest preoperative TMCs. On the other hand, patients with severe oligozoospermia and azoospermia showed a bad response. These findings are similar to the previously reported results (21). Also, we found that after varicocelectomy, the results of semen parameters correlated negatively with the duration of infertility which is in agreement with reported study (10).

In the present study, multivariate analysis revealed that varicocele grade effect on pregnancy rate was insignificant, which is in agreement with a previously published study (10). It was reported that varicocele size did not have an impact upon either semen improvement or pregnancy rate (22). In contrast, studies have shown that subclinical varicoceles are much more common, being present in $44 \%$ of fertile men and $60 \%$ of men attending infertility clinics (23). Despite the insignificant effect of varicocele size on pregnancy rate, we found that varicocele size had an adverse relationship with baseline semen parameters and direct relationship with potential improvement which is in agreement with previous findings (11).

In the present study, the difference in the postoperative TMCs between Groups I and III was statistically significant $(\mathrm{p}<0.05)$. The greatest increase in TMCs in patients with primary infertility 
was encountered in Group I and the pregnancy rate in this group was significantly higher than in Group III. Therefore, according to these results, we could assume that an increase in the TMCs after varicocelectomy is an indicator of pregnancy rate improvement. After varicocelectomy TMCs increased more than $18 \times 10^{6}$ in all our patients provided the preoperative TMCs were more than $5 \times 10^{6}$ and the pregnancy rate reached $34.4 \%$. These findings were comparable to previously reported results (24). Marmar et al. (24) according to their conducted meta-analysis study including only randomized, controlled trials concluded that varicocelectomy is an effective method for improving spontaneous pregnancy rates in the infertile male who has poor semen quality and palpable varicocele. On the other hand, many authors are still not convinced of the positive effect of varicocelectomy on pregnancy rate. There are many approaches for varicocelectomy, such as high ligation (retroperitoneal), inguinal, laparoscopic and subinguinal microscopic approach. The Subinguinal microscopic approach described by Marmar et al. (24) was considered as a gold standard. In our study most of our patients underwent inguinal varicocelectomy. Therefore, patients who were treated by other approaches were excluded from the study in order not to have an impact upon our results.

Through multivariate analysis, including patient's age, varicocele grade, and infertility period, we found that the infertility period was the only independent factor affecting sperm motility and pregnancy rate, which is in agreement with reported series (10).

\section{CONCLUSIONS}

Varicocele remains a risk factor for abnormal semen parameters that affect spontaneous pregnancy. Surgical varicocelectomy was found to improve the total sperm motility counts even in patients with azoospermia and to improve the spontaneous pregnancy rates especially in patients who have a total motile sperm count more than five million. The duration of infertility correlates negatively with the improvement in the total sperm count and the spontaneous pregnancy rates after varicocelectomy. Therefore, duration of infertility should be considerd in treating a patient with a varicocele as a cause of infertility.

\section{CONFLICT OF INTEREST}

None declared.

\section{REFERENCES}

1. Kolettis PN: Evaluation of the subfertile man. Am Fam Physician. 2003; 67: 2165-72. Erratum in: Am Fam Physician. 2003; 68: 1266.

2. No authors listed: The influence of varicocele on parameters of fertility in a large group of men presenting to infertility clinics. World Health Organization. Fertil Steril. 1992; 57: 1289-93.

3. Steckel J, Dicker AP, Goldstein M: Relationship between varicocele size and response to varicocelectomy. J Urol. 1993; 149: 769-71.

4. Hauser R, Paz G, Botchan A, Yogev L, Yavetz H: Varicocele: effect on sperm functions. Hum Reprod Update. 2001; 7: 482-5.

5. Marmar JL: The pathophysiology of varicoceles in the light of current molecular and genetic information. Hum Reprod Update. 2001; 7: 461-72.

6. Basar MM, Kisa U, Tuglu D, Kacmaz M, Basar H: The effect of varicocele on seminal plasma and serum inhibin-B levels in adolescent and adult men. Int Urol Nephrol. 2010; 42: 47-51.

7. Resorlu B, Kara C, Sahin E, Unsal A: The significance of age on success of surgery for patients with varicocele. Int Urol Nephrol. 2010; 42: 351-6.

8. Colpi GM, Carmignani L, Nerva F, Piediferro G, Castiglioni F, Grugnetti C, et al.: Surgical treatment of varicocele by a subinguinal approach combined with antegrade intraoperative sclerotherapy of venous vessels. BJU Int. 2006; 97: 142-5.

9. Cocuzza M, Cocuzza MA, Bragais FM, Agarwal A: The role of varicocele repair in the new era of assisted reproductive technology. Clinics (Sao Paulo). 2008; 63: 395-404.

10. Zorba UO, Sanli OM, Tezer M, Erdemir F, Shavakhabov S, Kadioglu A: Effect of infertility duration on postvaricocelectomy sperm counts and pregnancy rates. Urology. 2009; 73: 767-71.

11. Jarow JP: Effects of varicocele on male fertility. Hum Reprod Update. 2001; 7: 59-64. 
12. World Health Organization (WHO) Laboratory manual for the examination of human semen and spermcervical mucus interaction. Cambridge University Press, Cambridge 1992.

13. Cayan S, Erdemir F, Ozbey I, Turek PJ, Kadioglu A, Tellaloglu S: Can varicocelectomy significantly change the way couples use assisted reproductive technologies? J Urol. 2002; 167: 1749-52.

14. Sharma RK, Agarwal A: Role of reactive oxygen species in male infertility. Urology. 1996; 48: 835-50.

15. Zorgniotti AW, Macleod J: Studies in temperature, human semen quality, and varicocele. Fertil Steril. 1973; 24: 854-63.

16. Pintus C, Rodriguez Matas MJ, Manzoni C, Nanni L, Perrelli L: Varicocele in pediatric patients: comparative assessment of different therapeutic approaches. Urology. 2001; 57: 154-7.

17. Chehval MJ, Purcell MH: Deterioration of semen parameters over time in men with untreated varicocele: evidence of progressive testicular damage. Fertil Steril. 1992; 57: 174-7.

18. Saypol DC, Howards SS, Turner TT, Miller ED Jr: Influence of surgically induced varicocele on testicular blood flow, temperature, and histology in adult rats and dogs. J Clin Invest. 1981; 68: 39-45.
19. Haans LC, Laven JS, Mali WP, te Velde ER, Wensing CJ: Testis volumes, semen quality, and hormonal patterns in adolescents with and without a varicocele. Fertil Steril. 1991; 56: 731-6.

20. Sayfan J, Siplovich L, Koltun L, Benyamin N: Varicocele treatment in pubertal boys prevents testicular growth arrest. J Urol. 1997; 157: 1456-7.

21. Matkov TG, Zenni M, Sandlow J, Levine LA: Preoperative semen analysis as a predictor of seminal improvement following varicocelectomy. Fertil Steril. 2001; 75: 63-8.

22. Dubin L, Amelar RD: Varicocele size and results of varicocelectomy in selected subfertile men with varicocele. Fertil Steril. 1970; 21: 606-9.

23. Schlegel PN: Is assisted reproduction the optimal treatment for varicocele-associated male infertility? A cost-effectiveness analysis. Urology. 1997; 49: 83-90.

24. Marmar JL, Agarwal A, Prabakaran S, Agarwal R, Short RA, Benoff S, et al.: Reassessing the value of varicocelectomy as a treatment for male subfertility with a new meta-analysis. Fertil Steril. 2007; 88: 639-48.

\section{Correspondence address:}

Dr. Mohammed A Al-Ghazo

Associate professor of Urology

Department of General Surgery and Urology,

Faculty of Medicine

Jordan University of Science and Technology

PO Box 3030, Irbid, 22110, Jordan

Fax: + 96227200621

E-mail: alghazo@just.edu.jo
Submitted for publication:

January 05, 2011

Accepted after revision:

April 25, 2011 